\title{
Use of reliability tested clinically data towards a portable device measurement kit system for upper limb spasticity
}

\author{
Khairunnisa Johar a, Noor Ayuni Che Zakaria a, ${ }^{*}$, Fazah Akhtar Hanapiah ${ }^{b}$, Low Cheng Yee ${ }^{c}$,

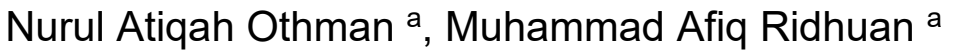 \\ ${ }^{a}$ Faculty of Mechanical Engineering, Universiti Teknologi MARA Shah Alam, Selangor, Malaysia \\ ${ }^{b}$ Faculty of Medicine, Universiti Teknologi MARA Selangor, 47000 Sungai Buloh, Malaysia \\ ${ }^{c}$ Faculty of Mechanical and Manufacturing Engineering, Universiti Tun Hussein Onn Malaysia, Parit Raja, Johor, Malaysia \\ * Corresponding author: ayuni8098@salam.uitm.edu.my
}

\section{Article history}

Received 11 Mac 2019

Revised 4 May 2019

Accepted 1 June 2019

Published Online 25 August 2019

\begin{abstract}
Spasticity refers to the abnormal symptom of having resistance in the joints when patients tried to make a movement. Patients with spasticity need to undergo multiple therapy sessions with medical intervention to ensure that the upper limb achieve maximal function. Modified Ashworth Scale (MAS) is frequently used in clinical assessment with grading on a scale. However, this scale is limited in sensitivity and the accuracy of this evaluation is dependent on the physician's and therapist's experience. This study suggests developing a portable measurement device kit system during clinical assessment to reduce inter- and intra-rater variability, and to assist clinicians in making quick clinical evaluation of spasticity. In this study, 19 patients were involved in the data acquisition. Assessment data from upper limb of patients with spasticity were recorded using a Manual Muscle Tester (MMT) and digital goniometer to measure force and the angular motion. During the assessment, patients were examined through slow and fast motion for spasticity evaluation. The collected data were analyzed to study intra-rater reliability value by using Statistical Package for the Social Sciences (SPSS). The results of Intraclass Correlation Coefficient (ICC) values for all patients were in range 0.78 to 0.89 . It can be considered that the collected data was reliable and can be used to formulate a model towards the development of a portable device measurement kit system for upper limb spasticity.
\end{abstract}

Keywords: Upper limb, stroke; rehabilitation

\section{INTRODUCTION}

Stroke is one of the biggest contributors to spasticity (Organization, 2004). Spasticity can be defined as hypertonia with several signs, which are the resistance to externally imposed movement that increases with increasing speed of stretch and varies with the direction of joint movement as well as the resistance to externally imposed movement that rapidly rises above a threshold speed or joint angle. From both signs mentioned, spasticity creates stiffness and tightness (Satkunam, 2003). The resistance is the reason why the muscles are unable to complete their Range of Motion (ROM) since it gives the sign of increased muscle tone (Adams \& Hicks, 2005). One side of human body will be weakened or paralyzed and needs time to recover from spasticity (Oujamaa et al., 2009). At this stage, the patients suffering from spasticity need rehabilitation to regain their normal movement. However, determining the efficacy of these management techniques depend on measurement tools that can objectively quantify spasticity. The quantitative assessment of spasticity is important for evaluation of potential effects of treatment interventions, with assessments of spasticity being challenged as having poor sensitivity and is not simple to execute (Silva et al., 2014).

From the review, past and current measurement devices are not suitable for everyday clinical use by physician to patient (refer Table
1). The goniometer is designed to measure the range of motion of the elbow joint (Kim et al., 2011). On the other hand, a manual muscle tester is used to measure the force given by the physician on the forearm of the subjects during a passive stretch motion. Other researcher also uses EMG sensor for force measurement.

There are technologies that have been shown to accurately measure spasticity, but they are often limited to laboratory-based research and are not suitable for everyday clinical use (Burridge et al., 2005). Clearly, there is a need for a clinically friendly tool that can improve spasticity assessment, which are clinically relevant for physicians. Thus, there is a gap to develop a measurement kit and a system that can enhance a physician's method of improving spasticity assessment. Generally, standardizing the level of spasticity is difficult to achieve since different physicians/clinicians lead to different evaluation findings (Biering-Sørensen et al., 2006). In which, the accuracy of this evaluation is depended on the physicians and clinicians' experience (Wu et al., 2006). Therefore, the physician/clinician needs a measuring device system to improve the rehabilitation process. In this paper, we present a conceptual design of a portable measurement device kit system and reliability test analysis of the input data from a single physician. 
Table 1 List of previous study of improved measurement device for spasticity assessment.

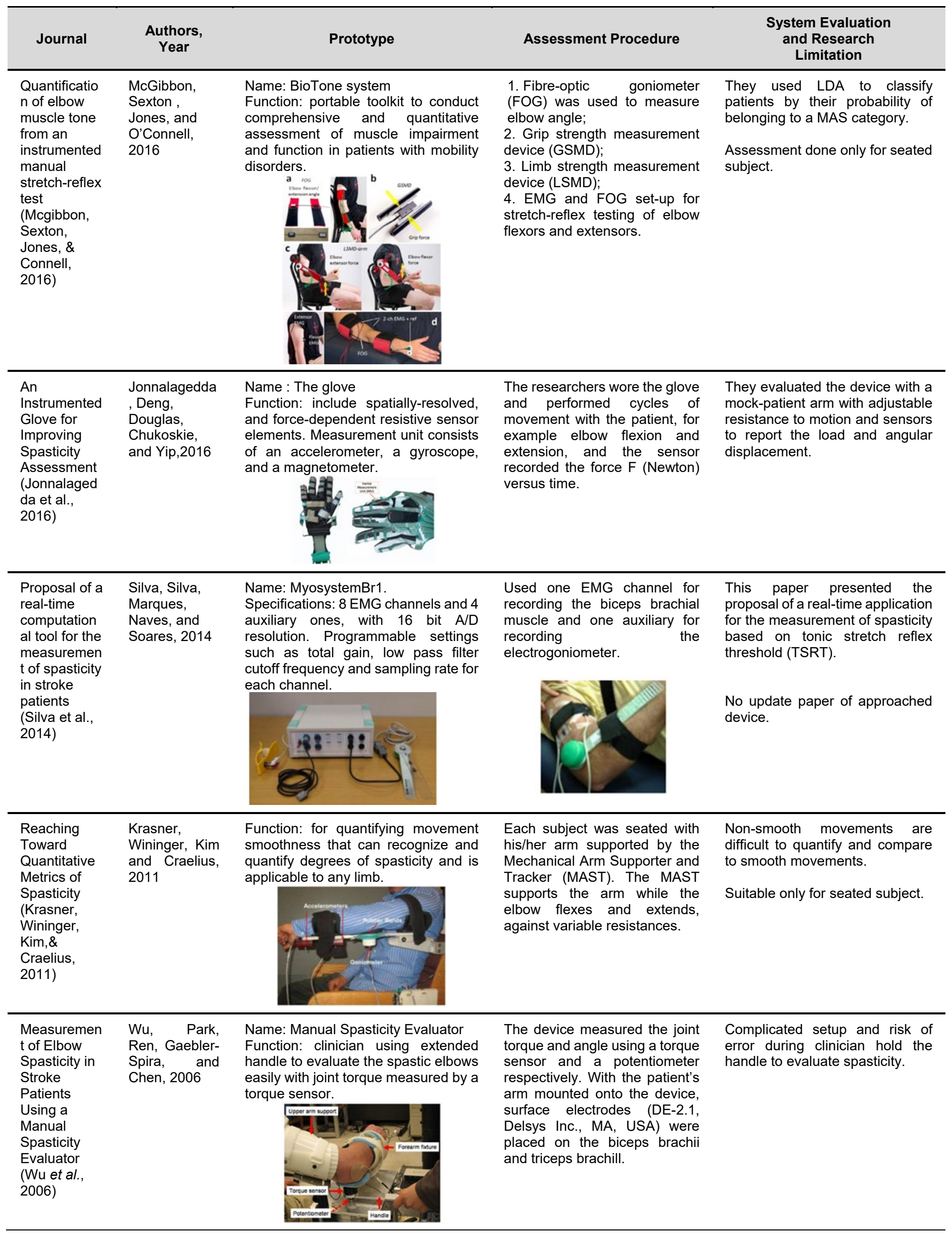




\section{CONCEPTUAL STUDY}

The purpose of this study was to develop a portable device measurement kit for improving spasticity assessment. In this portable device measurement kit, it includes a manual muscle tester (MMT), digital goniometer with sensor DAQ interface and laptop installed with evaluation system software (refer Fig 1). The developed system can evaluate the level of spasticity based on the Modified Ashworth Scale (MAS) in real time using neural network. The database will be saved from the classified data of spasticity during assessment. From this database, an algorithm for the system to be readable for identifying the level of spasticity was generated. At the end of the assessment, the neural network determined the level of spasticity according to quantifying the properties of spastic biceps muscle. Then, the measurement device measured the data in real time and translated the measurement data for spasticity evaluation in screen display. The outcome of this measurement device system was compared with our physician's evaluation to ensure the system was able to identify different levels of spasticity. The outcome may lead to improving the quality of post-stroke evaluation and thus the quality of post-stroke care.

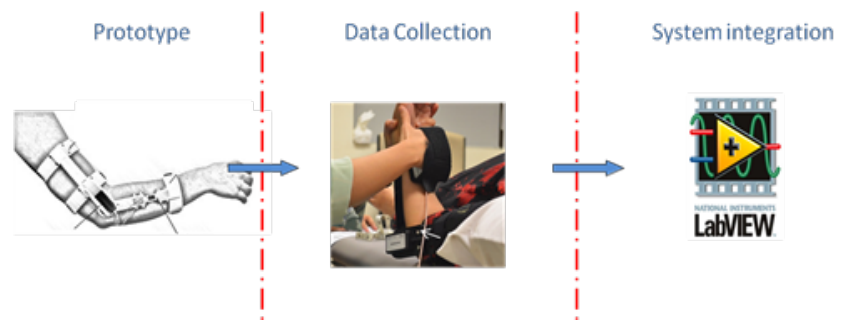

Fig 1 Conceptual idea of portable device measurement kit.

\section{METHODOLOGY}

The clinical data measurement was done by a professional rehabilitation physician in a government hospital and a university teaching centre in Malaysia. A total of 19 patients with spasticity were involved in the study. Each patient had different characteristics and levels of spasticity. Before conducting the clinical assessment, ethics approval was granted from UiTM Research Ethics Committee and the Medical Research Ethics Committee (MREC). The assessment was conducted with fast and slow passive motion of the elbow joint, where we measured the range of motion (ROM) and catch position of each patient (Zakaria et al., 2014). The goniometer (GNM-BTA from Vernier) was used to measure the angle of patient's arm movement and manual muscle tester from Sakaimed was used to measure the force.

Before the clinical procedures began, the goniometer was attached to the patient's arm and manual muscle tester placed to physician's palm. Both are connected to a Vernier SensorDAQ for data acquisition. The clinical procedures begin with slow motion through the range of movement. The physician stretched the forearm of the patient until reaching fully stretched position during slow extension. The estimated time for reaching fully stretched position was about 7 to 8 seconds and repeated three times. The clinical procedure continued with fast motion session. The physician stretched the patient's arm as fast as the patient can in order to obtain catch position. The estimated time to complete the fast motion session about 3 seconds and also repeated three times. Both motion sessions were recorded and the evaluation of level of spasticity determined by the physician.

\section{RESULTS AND DISCUSSION}

Standard Error Measurement (SEM) was used to obtain the error of measurement for the patients. To calculate the error happen during clinical procedure, the equation of Standard Error Measurement (SEM) is

$$
S E M=S D \times \sqrt{1-I C C}(1)
$$

The equation consists of standard deviation (SD) multiply with square root of sum of one minus Intraclass Correlation Coefficient (ICC) value. If the SEM values are below to one, the final answer will be same with SD value. If the SEM values is higher than 1, the SEM value will be equal to zero. Table 2 shows the Standard Error Measurement (SEM) value for slow and fast extension of the patients. Referring to Table 2, SEM values for the patients is equal to zero. Therefore, it can be assumed that the error measurements did not exist during the clinical procedure and the clinical data collected is valid and acceptable.

Table 2 SEM values for slow and fast extension of patient.

\begin{tabular}{ccccc}
\hline Patient & SD $_{\text {slow }}$ & SEM $_{\text {slow }}$ & SD $_{\text {fast }}$ & SEM $_{\text {fast }}$ \\
\hline $\mathbf{4}$ & 110.58 & 0 & 106.70 & 0 \\
$\mathbf{5}$ & 121.30 & 0 & 144.09 & 0 \\
$\mathbf{6}$ & 108.86 & 0 & 118.74 & 0 \\
$\mathbf{7}$ & 129.73 & 0 & 131.21 & 0 \\
$\mathbf{1 0}$ & 161.82 & 0 & 138.45 & 0 \\
$\mathbf{1 1}$ & 120.95 & 0 & 98.25 & 0 \\
$\mathbf{1 2}$ & 171.31 & 0 & 166.19 & 0 \\
$\mathbf{1 5}$ & 151.83 & 0 & 146.80 & 0 \\
$\mathbf{1 6}$ & 120.60 & 0 & 127.34 & 0 \\
$\mathbf{1 7}$ & 128.60 & 0 & 113.07 & 0 \\
$\mathbf{1 8}$ & 153.35 & 0 & 117.30 & 0 \\
$\mathbf{1 9}$ & 140.13 & 0 & 149.91 & 0 \\
\hline
\end{tabular}

The reliability test analysis is important towards this study to validate physician evaluation data as a database in our evaluation system. Intraclass Correlation Coefficient (ICC) is a descriptive statistic used when quantitative measurements are made on units that are organized into groups. To obtain the intra-rater reliability, the ICC was used during the data. It is used to calculate the consistency of measurements made by multiple observers measuring the same quantity. Table 3 shows the ICC value for slow and fast extension of the patients. From the table, the ICC values for slow and fast extension of patients shows good interpretation by referring to Koo and Li (2016). The ICC values for all patients ranged from 0.78 to 0.89 . The variables selected such as time taken, angle, and moment are in same quantity. Since all the patients showed good interpretation in intra-rater reliability test, this validated data can be used to formulate a system model towards the development of a portable device measurement kit system for upper limb spasticity.

Table 3 Intraclass Correlation Coefficient (ICC) values for patients.

\begin{tabular}{ccccc}
\hline Patient & ICC $_{\text {slow }}$ & Interpretation & ICC $_{\text {fast }}$ & Interpretation \\
\hline $\mathbf{4}$ & 0.86 & Good & 0.78 & Good \\
$\mathbf{5}$ & 0.85 & Good & 0.78 & Good \\
$\mathbf{6}$ & 0.87 & Good & 0.79 & Good \\
$\mathbf{7}$ & 0.85 & Good & 0.79 & Good \\
$\mathbf{1 0}$ & 0.85 & Good & 0.81 & Good \\
$\mathbf{1 1}$ & 0.89 & Good & 0.82 & Good \\
$\mathbf{1 2}$ & 0.84 & Good & 0.80 & Good \\
$\mathbf{1 5}$ & 0.85 & Good & 0.81 & Good \\
$\mathbf{1 6}$ & 0.89 & Good & 0.86 & Good \\
$\mathbf{1 7}$ & 0.89 & Good & 0.82 & Good \\
$\mathbf{1 8}$ & 0.86 & Good & 0.81 & Good \\
$\mathbf{1 9}$ & 0.88 & Good & 0.82 & Good \\
\hline
\end{tabular}

\section{CONCLUSION}

In conclusion, it shows that there are numerous assessment tools that can be used to measure spasticity. However, various issues are associated with these tools such as inconsistent psychometric properties and feasibility of using the measure in the clinical setting. In this work, previous study of improving measurement device was reviewed and a conceptual design based on reliable clinical data for the development 
of a portable measurement device kit approach has been presented. The limitation of the study was that there were several patients that have insufficient data. The incomplete data collected limited the researchers' ability to measure the intra-rater reliability. Further study needs to continue with more variable patient's data.

\section{ACKNOWLEDGEMENT}

The authors thank Universiti Teknologi MARA for funding the research work through the research grants [Ref. 600-IRMI/PERDANA 5/3 BESTARI (087/2018)]

\section{REFERENCES}

Adams, M. M., Hicks, A. L. (2005). Spasticity after spinal cord injury. Spinal Cord, 43(10), 577-586.

Biering-Sørensen, F., Nielsen, J. B., Klinge, K. (2006). Spasticity-assessment: A review. Spinal Cord, 44(12), 708-722.

Burridge, J. H., Wood, D. E., Hermens, H. J., Voerman, G. E., Johnson, G. R. van Wijck, F., ... Pandyan, a. D. (2005). Theoretical and methodological considerations in the measurement of spasticity. Disability and Rehabilitation, 27(1-2), 69-80.

Jonnalagedda, P., Deng, F., Douglas, K., Chukoskie, L., Yip, M., Ng, T. N., .. Garudadri, H. (2016). An instrumented glove for improving spasticity assessment. IEEE-NIH 2016 Special Topics Conference on Healthcare Innovations and Point-of-Care Technologies. November 9-11, Cancun, Mexico: 167-170.

Kim, K. S., Seo, J. H., Song, C. G. (2011). Portable measurement system for the objective evaluation of the spasticity of hemiplegic patients based on the tonic stretch reflex threshold. Medical Engineering and Physics, 33(1), 6269.

Koo, T. K., Li, M. Y. (2016). A guideline of selecting and reporting intraclass correlation coefficients for reliability research. Journal of Chiropractic Medicine, 15(2), 155-163.

Krasner, A., Wininger, M., Kim, N. H., Craelius, W. (2011). Reaching toward quantitative metrics of spasticity. 2011 IEEE 37th Annual Northeast Bioengineering Conference (NEBEC), 1-3 April, 2-3.

Mcgibbon, C. A., Sexton, A., Jones, M., Connell, C. O. (2016). Quantification of elbow muscle tone from an instrumented manual stretch-reflex test, 1(5), 1-11. Retrieved from https://www.oatext.com/Quantification-of-elbowmuscle-tone-from-an-instrumented-manual-stretch-reflex-test.php

World Health Organization (2004). Global burden of stroke, the atlas of heart disease and stroke. Geneva: World Health Organization. Retrieved from https://www.who.int/cardiovascular_diseases/resources/atlas/en/

Oujamaa, L., Relave, I., Froger, J., Mottet, D., Pelissier, J. Y. (2009). Rehabilitation of arm function after stroke. Literature review. Annals of Physical and Rehabilitation Medicine, 52(3), 269-293.

Satkunam, L. E. (2003). Rehabilitation medicine: 3. Management of adult spasticity. Canadian Medical Association Journal, 169(11), 1173-1179.

Silva, a. N., Silva, M. B., Marques, I. a., Naves, E. L. M., Soares, a. B. (2014). Proposal of a real-time computational tool for the measurement of spasticity in stroke patients. 5th ISSNIP-IEEE Biosignals and Biorobotics Conference (2014): Biosignals and Robotics for Better and Safer Living (BRC). 26-28 May. Salvador, Brazil.

Wu, Y. N., Park, H. S., Ren, Y., Gaebler-Spira, D., Chen, J. J., Zhang, L. Q. (2006). Measurement of elbow spasticity in stroke patients using a manual spasticity evaluator. 2006 International Conference of the IEEE Engineering in Medicine and Biology Society. 30 August-3 Sept. New York, USA: 3974-3977.

Zakaria, N. A. C., Low, C. Y., Hanapiah, F. A., Komeda, T., Inoue, K., Shazidi, M. S., Hamsan, H. M. (2014). Evaluation of upper limb spasticity towards the development of a high-fidelity part-task trainer. Procedia Technology, 15, $817-826$. 